\title{
Characterization of local Al-contacts by light beam induced current measurements and their verification by $2 \mathrm{D}$ simulation using flexPDE
}

\author{
Renate Horbeltt ${ }^{*, 1}$, Gabriel Micard', Philipp Keller', Giso Hahn' ${ }^{1}$, Reinhart Job ${ }^{2}$, and Barbara Terheiden ${ }^{1}$ \\ ${ }^{1}$ Department of Physics, University of Konstanz, 78457 Konstanz, Germany \\ ${ }^{2}$ Department of Electron Engineering and Computer Science, Münster University of Applied Sciences, 48565 Steinfurt, Germany
}

Keywords Al contacts, internal quantum efficiency, light beam induced currents, simulations, surface passivation, voids

*Corresponding author: e-mail renate.horbelt@uni-konstanz.de, Phone: +49 7531 884995, Fax: +49 7531883895

In recent years the relevance of passivated emitter and rear contact solar cells for industrial application increased due to significantly higher cell efficiencies compared to full area back surface field solar cells. However, the formation of local Al contacts is particularly sensitive to the manufacturing process parameters. Under non-optimized conditions, the eutectic in local Al contacts is missing and so-called voids are formed. So far, their impact onto the electrical parameters of the solar cells is not fully understood, mainly because of their difficult spatially resolved detection. Within this work the application of scanning acoustic microscopy circumvents this difficulty and allows a classification of local contacts in terms of "voids" or "filled contacts" on large cell area. The passivation quality of the BSF in the local contacts is investigated in detail through the local internal quantum efficiency (IQE) measured by light beam induced current (LBIC). It is found that there is a large spreading for the IQE values, attributed to a variation in BSF layer thickness. In addition, LBIC measurements of voids are fitted by $2 \mathrm{D}$ simulations, according to a detailed modelling of the surface recombination velocities
(SRV) in local contacts. The final model is based on a nonuniform SRV in the void's vicinity, attributed to laser damage close to the interface. It is demonstrated that the electrical parameters of the solar cell are affected by voids only when their surface is not sufficiently passivated by a local BSF.

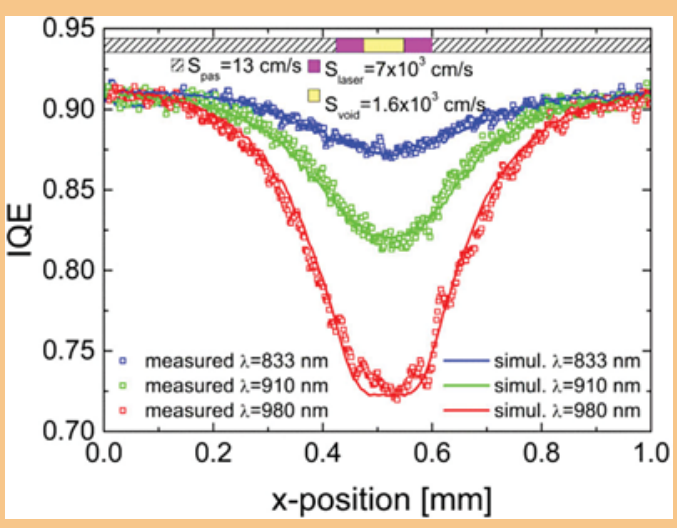

1 Introduction The alloying process of a full area aluminum back surface field (Al-BSF) based on screen printing technology was explained by Huster [1] according to the Al-Si binary phase diagram of Murray et al. [2]. However, local $\mathrm{Al}$ contact formation of passivated emitter and rear contact (PERC) solar cells differs from the full area case [3] in some aspects. One main difference is the diffusion of silicon away from the local contacts as investigated by several authors [4-6]. Under certain conditions local contacts without an eutectic layer between paste and silicon, so-called "voids", are formed. These "voids" were first characterized in detail by Urrejola et al. [4].

Since then many investigations have been carried out in order to prevent void formation as well as to understand the local contact formation in more detail. In this regard, the impact of contact geometry [7-11], paste composition [12-15], and firing conditions [16-18] have been studied. Other research activities were focusing on the electrical parameters, e.g., contact resistance or sheet resistivity [8, 19]. An additional topic of interest was the determination of 
surface recombination velocity (SRV) in local contacts [20] which is directly related to the BSF formation in local contacts [21].

The application of scanning electron microscopy (SEM) has been a common method to categorize contacts and voids in particular [22-24]. However, SEM imaging requires a high effort for sample preparation and the obtained crosssectional views give only little information concerning the spatial distribution of voids within a large area PERC solar cell. To circumvent these drawbacks, Dressler et al. [25] applied scanning acoustic microscopy (SAM). Combined with electroluminescence (EL) measurements it was demonstrated that a high amount of voids does not necessarily lead to a reduction in cell efficiency. This finding was confirmed by Horbelt et al. [18] explained by sufficiently thick BSF layers in voids.

Up to now, only few numerical simulations concerning passivation of local contacts have been carried out. These simulations demonstrate that a sufficiently thick local BSF layer is needed to achieve low values for SRV [22, 23, 26, 27]. This requirement is fulfilled in the case of filled contacts where in general a BSF layer with a thickness of several micrometres exists. In the case of a void, the BSF layer is thinner compared to a filled contact or completely missing [18].

Within this work, the BSF formed in local contacts is investigated in more detail by high resolution light beam induced current (LBIC) measurements revealing information about its passivation quality in filled contacts and in voids. In addition, a detailed modelling is used to interpret the observed experimental data.

2 Sample preparation For processing PERC solar cells, p-type Czochralski $(\mathrm{Cz}) \mathrm{Si}$ wafers $(2-3 \Omega \mathrm{cm}$, $125 \times 125 \mathrm{~mm}^{2}$, thickness around $\left.170 \mu \mathrm{m}\right)$ are used. A single side texturization step in alkaline solution is followed by wet chemical cleaning of the wafers. The homogeneous $\mathrm{n}^{+}$-emitter is formed by $\mathrm{POCl}_{3}$ diffusion in a quartz tube furnace, resulting in a sheet resistance of $60 \Omega \mathrm{sq}^{-1}$. After Pglass removal in HF solution, followed by wet chemical cleaning (Radio Cooperation of America, RCA) [28] a thermal oxidation in dry atmosphere is carried out. The thin oxide $(\sim 5 \mathrm{~nm})$ on the front side is covered by a hydrogenated $\mathrm{SiN}_{x}: \mathrm{H}$ layer via plasma-enhanced chemical vapor deposition (PECVD) with thickness of about $100 \mathrm{~nm}$. The layer serves as an etching barrier for the following wet chemical cleaning steps. The thermal oxide layer on the rear side is removed in HF solution. In a second step, the emitter on the rear side is etched in alkaline solution followed by wet chemical cleaning. In the end the final thickness of the $\mathrm{SiN}_{x}$ : $\mathrm{H}$ layer on the front is $\sim 70 \mathrm{~nm}$ acting as anti-reflection coating. The dielectric stack on the rear side of the PERC cells consists of a $10 \mathrm{~nm}$ thin $\mathrm{ALD} \mathrm{Al} \mathrm{O}_{3}$ layer (atomic layer deposition, Oxford FlexAL) covered by a $120 \mathrm{~nm}$ thick $\mathrm{SiN}_{x}: \mathrm{H}$ layer (remote PECVD). A picosecond pulsed laser (Coherent Inc., $532 \mathrm{~nm}$ wavelength, Gaussian profile) is used to form the local contact line openings on the rear side.
The contact opening width after laser ablation of the investigated cell area is $40 \mu \mathrm{m}$ with a constant pitch of $1 \mathrm{~mm}$. A commercially available silver paste is used for screen printing of the front side grid (finger width after firing $\sim 100 \mu \mathrm{m}$, pitch $=2.2 \mathrm{~mm}$ ). Note that the front side fingers are arranged perpendicular to the local rear contact openings in order to better separate the signals in the SAM and LBIC mappings. A commercially available $\mathrm{Al}$ paste is used on the rear side. After co-firing of the contacts in a belt furnace (centrotherm photovoltaics AG), edge isolation is carried out with a dicing saw (Disco DAD 3350).

\section{Experimental results and discussion}

3.1 IV-measurements The illuminated IV-curve is measured under standard conditions at a cell temperature of $25^{\circ} \mathrm{C}$ and 1 sun (AM1.5 global) illumination. The cell under investigation has a value of $652 \mathrm{mV}$ for the open circuit voltage $V_{\text {OC }}$, however it suffers from a low fill factor FF of $76.2 \%$ and a low short circuit current density $j_{\mathrm{SC}}$ of $36.5 \mathrm{~mA} \mathrm{~cm}^{-2}$, ending up in a cell efficiency $\eta$ of $18.2 \%$.

3.2 Definition of voids The local contacts shown in Fig. 1 are from the cell described above. The contact opening width after laser ablation was $40 \mu \mathrm{m}$, as indicated in Fig. 1a. Due to the dissolution of silicon during contact formation, the width increases up to $\sim 70 \mu \mathrm{m}$ after firing.

Figure 1a and $\mathrm{b}$ show the SEM images (Zeiss Neon 40 EsB), taken with different detectors, of the same "filled contact" with an eutectic between the Al-paste and the Si bulk. Figure 1a was taken with the Inlens detector (detecting from above the sample in the column), which is more suitable for detecting a contrast in doping concentration, i.e., a BSF layer in the local contacts. Due to the higher doping concentration, a BSF layer appears as bright region in this

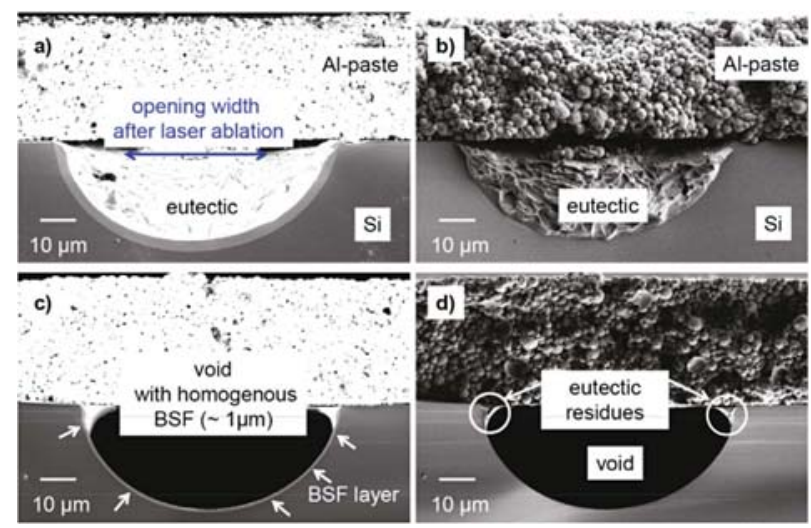

Figure 1 Classification of local contacts. Figure 1a and b show the same "filled contact," characterized by an eutectic between Alpaste and silicon. This eutectic layer is missing in a "void" (Fig. 1c and d). Both contacts show a homogeneous BSF formation. The figures on the left are taken with the Inlens detector of the SEM. The BSF appears as bright region. The figures on the right are with the SE2 detector emphasizing the morphology of a local contact, BSF layers are not detectable. 
image. The contrast is caused by Si ionization energy, which depends on doping concentration [29]. To intensify the contrast, the image is overexposed. Focusing on the other hand on the morphology of a local contact, the application of the SE2 detector (detecting from inside the chamber declined to the sample's surface normal) is beneficial (Fig. 1b). However, then the BSF is not detectable.

In contrast to a "filled contact" the eutectic is missing in a "void." Figure 1c exemplifies a void with homogeneous BSF formation. Applying the SE2 detector at the same contact reveals that there is a small area of eutectic composition detectable at the edges of the contact (marked in white in Fig. 1d). It has to be pointed out that voids show in general a thinner BSF compared to a filled contact. In some voids a BSF layer is completely missing [18].

3.3 Spatially resolved detection of voids The scanning acoustic microscope (KSI 400, Kraemer Sonic Industries) allows the spatially resolved and non-destructive detection of voids in local Al contacts. It is operating with a maximum scan speed of $2000 \mathrm{~mm} \mathrm{~s}^{-1}$ and a maximum lateral resolution of $10 \mu \mathrm{m}$.

The measurement principle is based on the "pulse-echomode": a transducer converts an electrical signal into an acoustic signal which is focused and emitted. The solar cell under investigation is mounted on a chuck and submerged by deionized water which serves as a coupling medium for the ultrasonic waves. Due to different materials and surfaces within the solar cell, the incoming signal is reflected and scattered. Only the reflected part of the signal is detected by the transducer and converted back to an electrical one. By analyzing the amplitude, phase, and time of flight, a pixelby-pixel image of the scanned area is created. Voids in the local $\mathrm{Al}$ contacts appear as dark lines in the image.

Figure 2 shows a high resolution scan with an area of $12 \times 12 \mathrm{~mm}^{2}$. The dark bar on the right-hand side is the front side busbar, the horizontal lines indicate the front side fingers no. 40-44. The vertical lines are the local contacts on the rear side, serially numbered from 1 to 10 . Obviously contacts $1,4,5,7$, and 9 are voids over the complete scanned area. Contact 8 is a filled contact within the investigated area. All other contact lines show some fractions of filled contacts as well as voids within one local contact line.

3.4 Internal quantum efficiency at local contacts For LBIC measurements an in-house built setup is used [30]. The solar cell is mounted on a movable $x y$ stage, resulting in a spatially resolved mapping of the short circuit current which is locally generated by a laser spot. Three different lasers with wavelengths of 833, 910, and $980 \mathrm{~nm}$ are applied, corresponding to a penetration depth of the monochromatic light of about 15,36 , and $103 \mu \mathrm{m}$ in $\mathrm{Si}$, respectively [31]. By calibrating the light intensity with a reference cell and measuring the reflected fraction of the incident light, combined with a reflection calibration the spatially resolved internal quantum efficiency (IQE) map is generated. Due to the variation of penetration depths of the

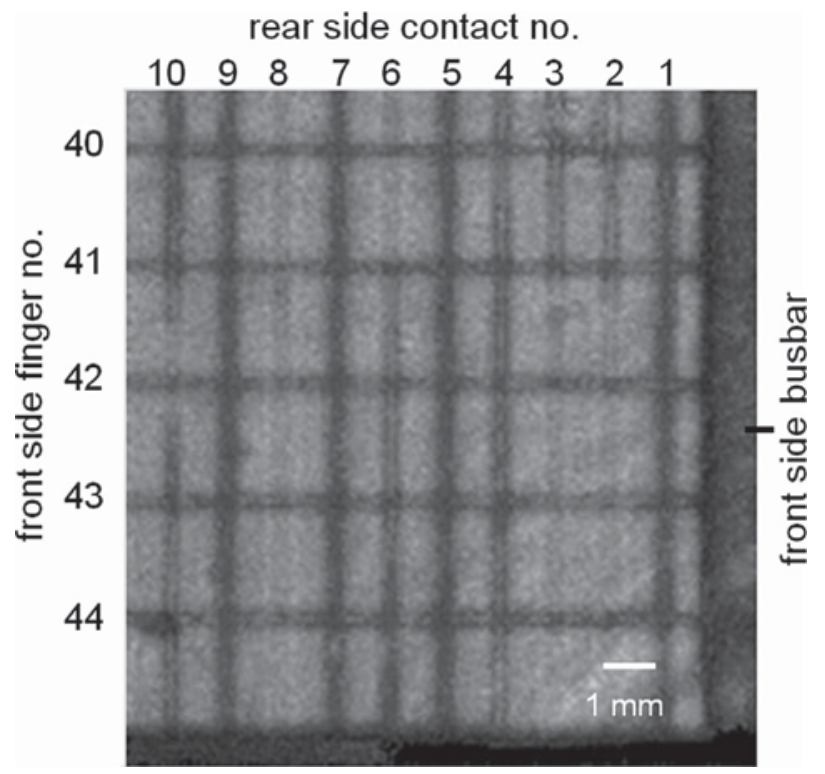

Figure 2 Image of the scanning acoustic microscope measurement. The scanned cell area is $12 \times 12 \mathrm{~mm}^{2}$. The bar on the righthand side is the front side busbar, the horizontal lines indicate the front side fingers. The local rear contacts run vertically. Within the local contacts dark lines correspond to voids whereas brighter lines stand for filled contacts.

incoming laser beams, information from different depths in the solar cell is obtained. Because of its higher penetration, results for $980 \mathrm{~nm}$ wavelength are of specific interest revealing the impact of a BSF on the passivation quality at the local rear contacts. Hence, the results discussed are restricted to the laser with a wavelength of $980 \mathrm{~nm}$. The results of the less penetrative lasers are only used for the 2D simulations in Section 3.5.

Figure 3 shows an IQE mapping of an area of $12 \times 12 \mathrm{~mm}^{2}$ of a solar cell. The high resolution of the mapping is achieved by working with a laser beam diameter of $\sim 10 \mu \mathrm{m}$ and a step width of $10 \mu \mathrm{m}$ during scanning. The scanned area corresponds to that in Fig. 2 which allows a correlation of the contact structure (void or filled contact) to the LBIC signal. An IQE value between 0.88 and 0.93 for the passivated areas in between the local contacts allows distinguishing these areas from the local contact areas, despite the fact that the rear side passivation is not at its possible optimum.

For a more detailed characterization of the local contacts an even higher resolution of the IQE mapping (step width of $2 \mu \mathrm{m})$ is taken. The investigated areas $\left(1 \times 2 \mathrm{~mm}^{2}\right)$ of contact no. $1,2,4$, and 9 are marked in red in Fig. 3 . The resulting high resolution mappings are shown in Fig. 4. Note the different scaling of the four individual IQE measurements.

Focusing on the voids (contact no. 1, 4, 9) it becomes obvious that there is a large variation of IQE values. While contact no. 9 shows a very low IQE of 0.72 , contact no. 4 features a significantly higher value of 0.88 . The IQE of contact no. 1 varies within the area of interest from top to 


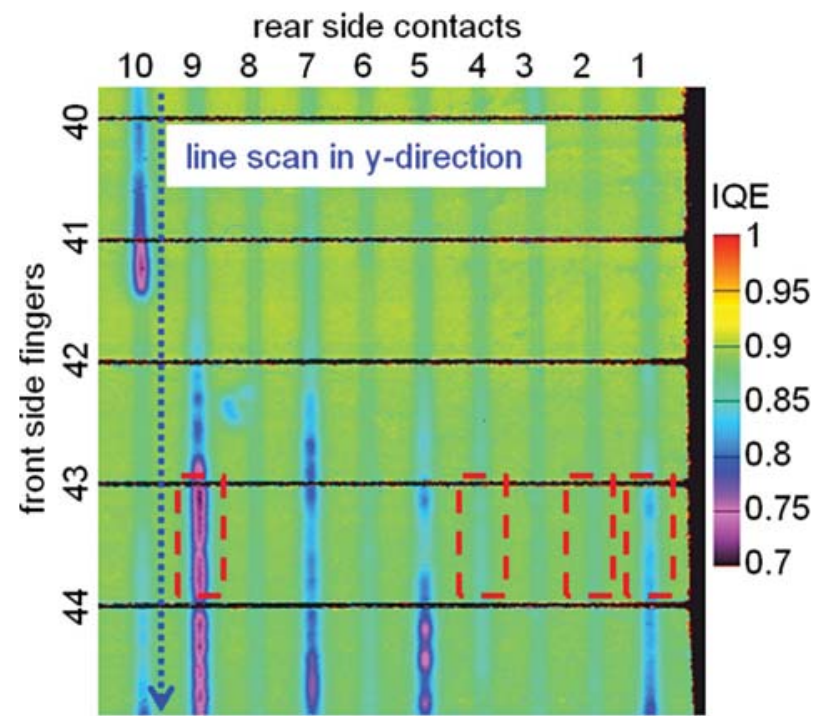

Figure $3 \mathrm{IQE}$ mapping of a cell area of $12 \times 12 \mathrm{~mm}^{2}$ at $980 \mathrm{~nm}$ ) corresponding to Fig. 2. The areas marked in red are investigated in more detail by high resolution mappings (see Fig. 4). The blue arrow indicates the direction of the $y$-line scan of rear side contact no. 10, shown in Fig. 6.

bottom of the image. In the upper part of the void there is a low IQE value of 0.72 comparable to that of contact no. 9. In the lower part the IQE increases noticeable up to 0.8 .

The wide range of IQE values for voids is attributed to a strong variation in the BSF layer thickness. These results confirm the findings of Lin et al. [32] expecting a high SRV for an inadequate LBSF formation.

Contact no. 2 - indicated as a filled contact by SAM measurements in Fig. 2 - reveals an IQE nearly as high as that of the passivated areas. Hence, the BSF in the filled contacts leads to a low SRV of the local contact.

The red arrows in Fig. 4 illustrate the positions where a line scan in $x$-direction is carried out ( $x$-line scan). The IQE value of each pixel is plotted as a function of position. Figure 5 reveals the corresponding results.

At contact no. 1 two $x$-line scans were carried out, one at position 1 with high IQE value and an additional one at
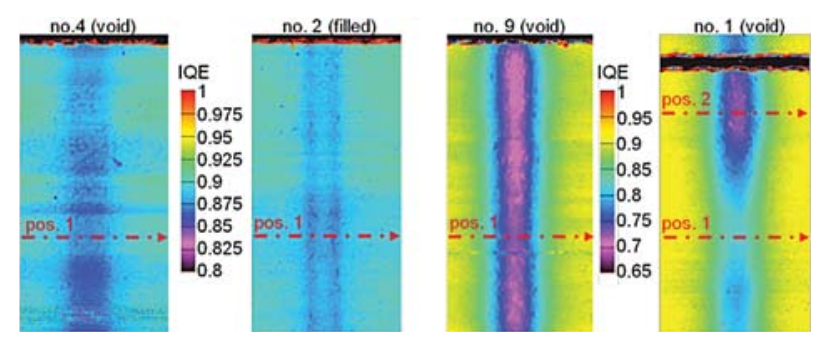

Figure 4 High resolution IQE mappings (at $980 \mathrm{~nm}$ ) of four rear contacts $\left(1 \times 2 \mathrm{~mm}^{2}\right)$. Note the different scaling of the IQE values. The dark lines in the upper part of the images indicate front side finger no. 43. The red lines (pos. 1 and 2) indicate the position where a line scan in $x$-direction is carried out.

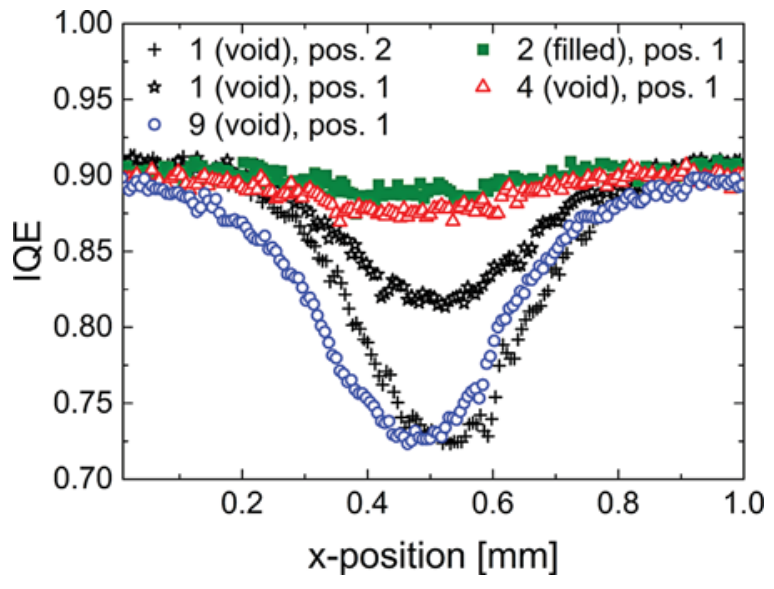

Figure 5 Line scans in $x$-direction of different local contacts as indicated in Fig. 4. Contact No. 1 was investigated at two different positions (pos. 1 and 2), the other contacts were investigated at pos. 1 .

position 2 with low IQE value in order to visualize the considerable variation along a very limited contact length. The other results are achieved at position 1 .

The $x$-line scans exemplify the large variation in IQE for the selected contacts. Contact no. 1 (void) at position 2 has an IQE value as low as that of contact no. 9 (void). However, there is a void (contact no. 4) which is nearly on the same high IQE level than a filled contact (no. 2). These two contacts show only a very low drop in IQE across the contact area.

To highlight the strong variation within one local Al contact, a line scan in $y$-direction is carried out for contact no. 10, as indicated in Fig. 3. According to SAM measurements shown in Fig. 2 it is a contact which is only filled nearby front side finger 42 . The rest of the contact is characterized as a void. The result of the $y$-line scan is shown in Fig. 6.

The numbers marked in blue indicate signal drops caused by the corresponding front side finger. The starting point of the IQE is 0.83 at $y$-position 0 . The IQE declines down to 0.73 at $y$-position $3.8 \mathrm{~mm}$, followed by a noticeable increase up to 0.88 . At this position a void ends and the local contact is filled (see Fig. 2). The IQE data points of the positions where the contact is filled are marked in green. However, the filled fraction ends closely after front side finger 42. Nevertheless, the IQE continues on the high value, due to a low SRV-value of the void, i.e., indicating a sufficiently thick local BSF layer there.

To quantify the SRV in the different regions of the voids, 2D simulations of the IQE of the solar cell are carried out as described in the following section.

3.5 2D simulation of rear contact It was demonstrated by Micard [33, 34] that the simulation software flexPDE [35] is an appropriate tool to solve the partial differential equation to investigate the recombination mechanisms on grain boundaries in multicrystalline silicon. 


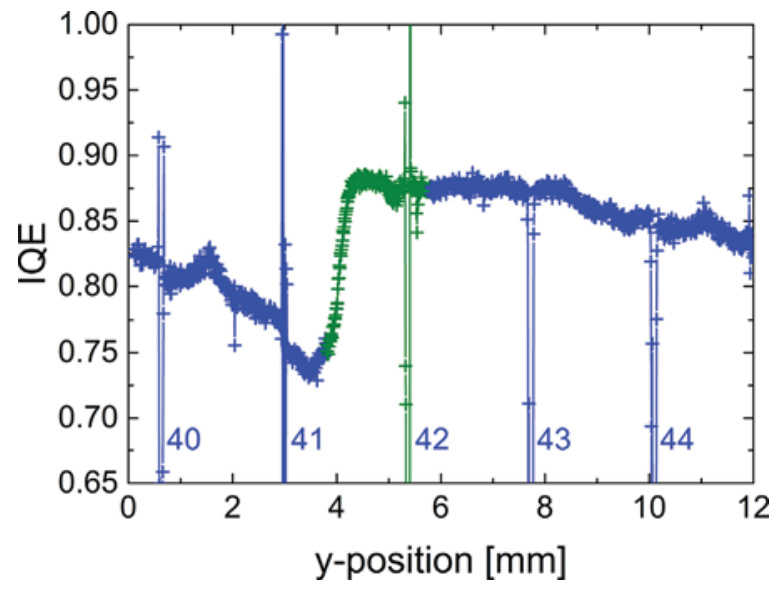

Figure 6 Scan in $y$-direction of contact no. 10 as indicated in Fig. 4. The numbers indicate a signal drop caused by the corresponding front side finger. The green marked data points indicate the area of the local contact which features an eutectic within the contact.

Micard carried out his studies fitting LBIC linescans by solving the partial differential equation of the minority carrier diffusion. As a high contrast to noise ratio is required for a reliable fitting procedure, the application of this routine to the measurements shown above is restricted to large signal variations as detected in voids with high SRV only. In the following, the results of the LBIC mapping at contact no. 9 (void) are compared with finite element simulations performed by flexPDE in order to determine the SRV within this void. Based on the estimated SRV, a conclusion about the passivation quality of the BSF can be drawn.

Moving Gaussian laser beams of different wavelengths used in our LBIC setup $(833,910$, and $980 \mathrm{~nm})$ generate charge carriers in the silicon bulk (thickness of $170 \mu \mathrm{m}$, bulk lifetime of $200 \mu \mathrm{s}$ ) that are collected at the junction (the LBIC signal) or recombine in the volume or at recombinative surfaces.

The minority carrier distribution is obtained by solving the minority carrier diffusion equation in steady state expressed as

$$
\Delta n\left(r_{\mathrm{G}}\right)-\frac{n\left(r_{\mathrm{G}}\right)}{L_{\mathrm{n}}^{2}}=-\frac{-G\left(r_{\mathrm{G}}\right)}{D_{\mathrm{n}}},
$$

with $\Delta$ the Laplace operator, $n$ the excess carrier density of the minorities, $r_{\mathrm{G}}$ the generalized coordinate of the position which would be expressed by $x, y$, and $z$ in 3D Cartesian coordinates [33]. $G$ is the generation rate distribution of charge carriers, $L_{\mathrm{n}}$ the diffusion length of electrons, and $D_{\mathrm{n}}$ the diffusion constant for electrons.

At the top surface the boundary condition $n(0)=0$ is imposed corresponding to the junction in short circuit condition [33]. The bottom surface is divided in different parts corresponding to the void and passivated surface outside that are both described by a recombinative boundary condition of the following form:

$$
\left.n\left(r_{\mathrm{G}}\right)\right|_{\Sigma} S_{\mathrm{n}}=-\left.D_{\mathrm{n}} \nabla_{\mathrm{n}} \cdot \vec{n}\right|_{\Sigma}
$$

introducing $S_{\mathrm{n}}$ the surface recombination velocity of electrons at the surface $\sum, \nabla$ the Del operator and $n$ the unit vector normal to the surface oriented [33].

The collection of charge carriers generated by the two less penetrative lasers (833 and $910 \mathrm{~nm}$ ) is affected by recombination in the bulk mainly and optical effects of the rear side of the solar cell do not influence the generation rate distribution. Hence, the accurate fitting of these laser profiles is a prerequisite for the consistency of the applied model.

We set the SRV of the passivated areas between the local Al contacts $\left(S_{\mathrm{pas}}\right)$ to $13 \mathrm{~cm} \mathrm{~s}^{-1}$ and the contact width to $70 \mu \mathrm{m}$ in agreement with the contact width determined by SEM after contact formation.

In a first attempt the best fit is obtained considering a homogeneous surface recombination velocity of $S_{\text {void }}=2$ $\times 10^{4} \mathrm{~cm} \mathrm{~s}^{-1}$. This value is in good agreement with SENTAURUS numerical simulations by Chen et al. [23]. The results of the LBIC measurements and the corresponding simulation are shown in Fig. 7.

The simulations fit the measurements well for the lasers with wavelengths of 833 and $910 \mathrm{~nm}$, demonstrating the consistency of the model in the top and middle part of the bulk. However, the simulation for the wavelength of $980 \mathrm{~nm}$ leads to a lower IQE value than the measured one in the center of the contact. Additionally, the walls of the LBIC profile are not correctly matched, indicated by a higher IQE value of the simulated profile compared to the measured one. This contrary behavior of the IQE value within the contact area cannot be explained by optical effects. To correct the fit of the walls, $S_{\text {void }}$ is increased up to $2 \times 10^{5} \mathrm{~cm} \mathrm{~s}^{-1}$ (Fig. 7). Now, the walls of the LBIC profile of the $980 \mathrm{~nm}$ laser are matched well. However, the

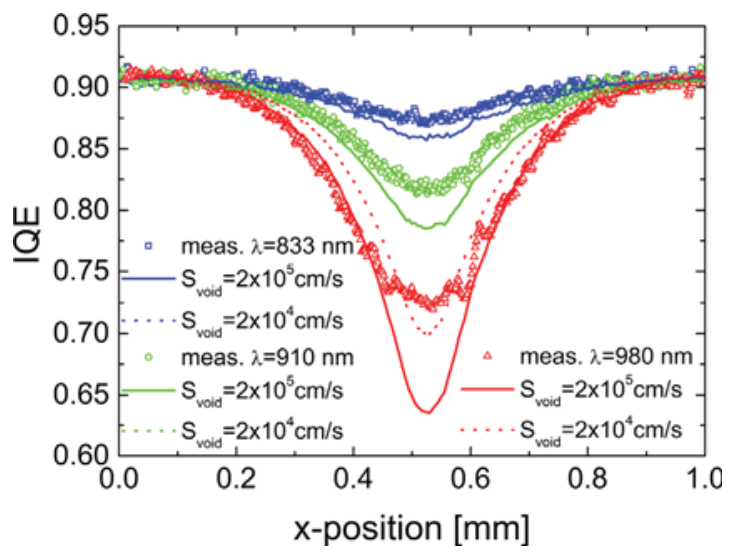

Figure 7 IQE measurement of a contact profile for lasers at $\lambda=833,910$, and $980 \mathrm{~nm}$ with simulated profiles considering the void is a $70 \mu \mathrm{m}$ region at the back with an homogeneous SRV in the void with $S_{\text {void }}=2 \times 10^{4} \mathrm{~cm} \mathrm{~s}^{-1}$ and $S_{\text {void }}=2 \times 10^{5} \mathrm{~cm} \mathrm{~s}^{-1}$. 
deviation of the simulated to the measured values for the two less penetrative lasers indicates the invalidity of the applied model. In addition, the bottom part of the bulk seems to be inadequately described by the assumption of a constant SRV in the entire void: both simulations of the $980 \mathrm{~nm}$ laser show a lower value for the simulated IQE than for the measured one.

For a further improvement of the fit, we consider that the void is surrounded by a laser damaged area on each side. The application of a Gaussian laser beam opens the dielectric layer locally in its center where the pulse energy is sufficiently high for a complete ablation. On the other hand, the pulse hitting the dielectric next to the ablated area results in a decline of the passivation quality and a damage of the silicon bulk beneath the dielectric layer while the dielectric still remains on the wafer surface.

Taking these facts into account, the input parameters for the simulation were adapted as follows: the SRV of the void itself was set to a value of $S_{\text {void }}=1.6 \times 10^{3} \mathrm{~cm} \mathrm{~s}^{-1}$, maintaining the aforementioned width of $70 \mu \mathrm{m}$, an area with a width of $50 \mu \mathrm{m}$ was added on each side of the void with an SRV of $S_{\text {laser }}=7 \times 10^{3} \mathrm{~cm} \mathrm{~s}^{-1}$. These parameters led to a precise simulation of the less penetrative lasers (Fig. 8), demonstrating the validity of the adapted model. Focusing on the $980 \mathrm{~nm}$ laser reveals a good agreement of the simulated IQE value with the measured one in the center of the contact, too.

In order to evaluate the scope of this adapted model, an additional simulation was carried out, increasing the width of the laser damaged area to $60 \mu \mathrm{m}$ on each side. This induced a decrease of the SRV in the laser damaged areas to $S_{\text {laser }}$ to $4.6 \times 10^{3} \mathrm{~cm} \mathrm{~s}^{-1}$ to match the walls of the LBIC profile dip and an increase of the SRV of the void to a value of $S_{\text {void }}=2.1 \times 10^{3} \mathrm{~cm} \mathrm{~s}^{-1}$ to match the bottom of the LBIC profile. This parameter set leads also to a correct fit of all three laser wavelengths which gives a hint about the uncertainty associated with such LBIC fit.

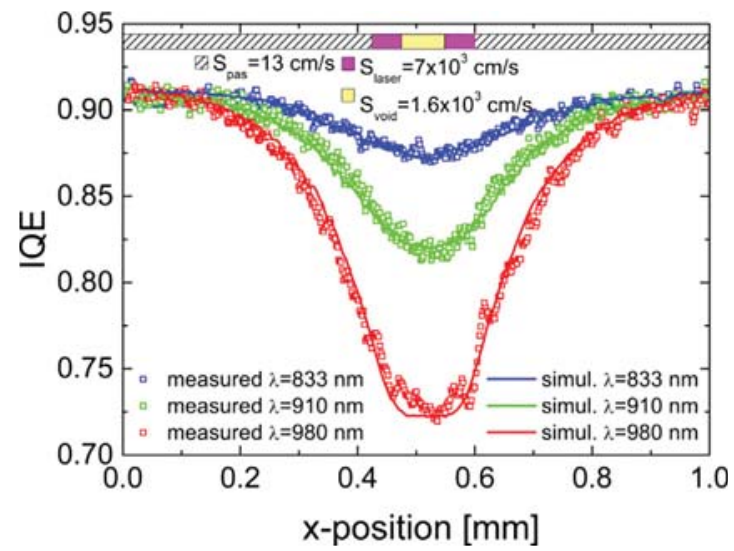

Figure 8 IQE measurement of an IQE profile for lasers at $\lambda=833$, 910 , and $980 \mathrm{~nm}$ with simulated profiles considering the void is a $70 \mu \mathrm{m}$ region at the back with an SRV $S_{\text {void }}=1.6 \times 10^{3} \mathrm{~cm} \mathrm{~s}^{-1}$ surrounded by two laser damaged areas of $50 \mu \mathrm{m}$ each with SRV $S_{\text {laser }}=7 \times 10^{3} \mathrm{~cm} \mathrm{~s}^{-1}$.
Hence, the applicability of the adapted model, based on a non-uniform SRV in the void's zone, is demonstrated.

4 Conclusions Combining scanning acoustic microscopy (SAM) measurements and LBIC measurements permits a more detailed knowledge of the electrical characteristics of local $\mathrm{Al}$ contacts in general, and voids in particular compared to the standard combination of SAM and electroluminescence. A large variation of IQE values was demonstrated.

To understand the differences in IQE values in more detail, the surface recombination velocity SRV of a void was determined from 2D simulations. Assuming one constant SRV value for the width of the void does not match the LBIC $x$-line scan appropriately. However, when taking into account an additional 50-60 $\mu \mathrm{m}$-wide laser damaged zone parallel to the contact, the $x$-line scan and the simulation could be brought into agreement. In this way, the SRV in the void was determined to $\sim 2 \times 10^{3} \mathrm{~cm} \mathrm{~s}^{-1}$ and in the laserdamaged zone to $5-7 \times 10^{3} \mathrm{~cm} \mathrm{~s}^{-1}$.

These effects are attributed to the laser ablation process of the dielectric layer. In the center of the Gaussian laser profile the dielectric layer is completely ablated. A decline of the pulse energy towards the edge of the laser spot damages the silicon bulk while the dielectric layer still remains on the surface. With this combination of LBIC, SAM, and simulation a detailed investigation of local PERC contacts is possible, significantly supporting the development of high efficiency PERC-type solar cells.

Acknowledgments The authors would like to thank J. Engelhardt, A. Herguth, L. Mahlstaedt, F. Mutter for their support. The financial support for parts of this work by the German BMU under contract FKZ 0325581 is gratefully acknowledged.

\section{References}

[1] F. Huster, in: Proc. 20th EUPVSEC, Barcelona, Spain (2005), p. 1466.

[2] J. L. Murray and A. J. McAlister, Bull. Alloy Phase Diagrams 74-84 (1984), DOI: 10.1007/BF02868729.

[3] F.S. Grasso, L. Gautero, J. Rentsch, R. Preu, and R. Lanzafame, in: Proc. 2nd Workshop on Metallization for Crystalline Silicon Solar Cells, Konstanz, Germany (2010), p. 15.

[4] E. Urrejola, K. Peter, H. Plagwitz, and G. Schubert, Appl. Phys. Lett. 98, 153508 (2011), DOI: 10.1063/1.3579541.

[5] T. Lauermann, B. Fröhlich, G. Hahn, and B. Terheiden, Prog. Photovolt. 23, 10-18 (2015), DOI: 10.1002/pip.2388.

[6] J. Müller, K. Bothe, S. Gatz, and R. Brendel, Phys. Status Solidi RRL 6, 111-113 (2012), DOI: 10.1002/pssr.201105611.

[7] T. Lauermann, A. Zuschlag, S. Scholz, G. Hahn, and B. Terheiden, in: Proc. 26th EU PVSEC, Hamburg, Germany (2011), p. 1137.

[8] E. Urrejola, K. Peter, H. Plagwitz, and G. Schubert, J. Appl. Phys. 98, 153508 (2011), DOI: 10.1063/1.3579541.

[9] M. Bähr, G. Heinrich, O. Doll, I. Köhler, C. Maier, and A. Lawerenz, in: Proc. 26th EU PVSEC, Hamburg, Germany (2011), p. 1203. 
[10] M. Rauer, C. Schmiga, R. Woehl, K. Rühle, M. Hermle, M. Hörteis, D. Biro, and S. W. Glunz, IEEE. J. Photovolt. 1, 22-28 (2011), DOI: 10.1109/JPHOTOV.2011.2161864.

[11] J. Müller, S. Gatz, K. Bothe, and R. Brendel, in: Proc. 38th IEEE PVSC, Austin, USA (2012), p. 2223.

[12] M. Rauer, R. Woehl, C. Schmiga, M. Hermle, M. Hörteis, and D. Biro, IEEE Electron Dev. Lett. 32, 916-918 (2011), DOI: 10.1109/LED.2011.2143385.

[13] K. Wijekoon, H. Khatri, D. Tanner, L. Zhang, A. Shaikh, and H. Ponnekanti, in: Proc. 27th EU PVSEC, Frankfurt, Germany (2012), p. 608.

[14] V. Meemongkolkiat, K. Nakayashiki, D.S. Kim, S. Kim, A. Shaikh, A. Kuebelbeck, W. Stockum, and A. Rohatgi, in: Proc. 4th WC PEC, Waikoloa, USA (2006), p. 1338.

[15] M. Balucani, L. Serenelli, K. Kholostov, P. Nenzi, M. Miliciani, F. Mura, M. Izzi., and M. Tucci, Energ. Procedia 43, 100-110 (2013), DOI: 10.1016/j.egypro.2013.11.094.

[16] E. Urrejola, K. Peter, H. Plagwitz, and G. Schubert, J. Appl. Phys. 110, 056104 (2011), DOI: 10.1063/1.3633510.

[17] T. Fang, C.M. Lin, K.J. Li, L.T. Wang, and W.C. Tang, in: Proc. 26th EU PVSEC, Hamburg, Germany (2011), p. 2220.

[18] R. Horbelt, A. Herguth, G. Hahn, R. Job, and B. Terheiden, in: Proc. 29th EU PVSEC, Amsterdam, Netherlands (2014), p. 427.

[19] J. Krause, R. Woehl, M. Rauer, C. Schmiga, J. Wilde, and D. Biro, Sol. Energ. Mater. Sol. Cells 95, 2151-2160 (2011), DOI: 10.1016/j.solmat.2011.03.017.

[20] J. Müller, K. Bothe, S. Gatz, H. Plagwitz, G. Schubert, and R. Brendel, Energ. Procedia 8, 337-342 (2011), DOI: 10.1016/j. egypro.2011.06.146.

[21] P. Lölgen, Surface and Volume Recombination in Silicon Solar Cells, PhD Thesis, University of Utrecht (1995), ISBN: 90-393-0548-X.

[22] D. Chen, Y. Yang, Z. Li, Z. Liang, Z. Feng, P. Verlinden, and H. Shen, in: Proc. 27th EU PVSEC, Frankfurt, Germany (2012), p. 1303.
[23] Y. Chen, P. Altermatt, J. Dong, S. Zhang, J. Liu, D. Chen, W. Deng, Y. Jiang, B. Liu, W. Xiao, H. Zhu, H. Chen, H. Jiao, X. Pan, M. Zhong, D. Wang, J. Sheng, Y. Zhang, H. Shen, Z. Feng, and P.J. Verlinden, in: Proc. 40th IEEE PVSC, Colorado, USA (2014), p. 3322.

[24] S. Großer, R. Mayerhofer, and J. Theobald, in: Proc. 29th EU PVSEC, Amsterdam, Netherlands (2014), p. 1395.

[25] K. Dressler, S. Dauwe, T. Droste, J. Rossa, R. Meidel, K. Schünemann, K. Ramspeck, Y. Gassenbauer, and A. Metz, in: Proc. 27th EU PVSEC, Frankfurt, Germany (2012), p. 755.

[26] Y. Chen, H. Shen, and P. Altermatt, Sol. Energy Mater. Sol. Cells 120, 356-362 (2014), DOI: org/10.1016/j. solmat.2013.05.051.

[27] S. Gatz, K. Bothe, J. Müller, T. Dullweber, and R. Brendel, Energ. Procedia 8, 318-323 (2011), DOI: 10.1016/j. egypro.2011.06.143.

[28] W. Kern and D. A. Puotinen, RCA Rev. 3, 187 (1970).

[29] C. P. Sealy, M. R. Castell, and P. R. Wilshaw, J. Electron Micros. 49, 311 (2000).

[30] T. Pernau, M. Spiegel, P. Fath, and E. Bucher, in: Proc. 17th EU PVSEC, München, Germany (2001), p. 702.

[31] M. A. Green and M. Keevers, Prog. Photovolt. 3, 189-192 (1995), DOI: 10.1002/pip.4670030303.

[32] D. Lin, M. Abbott, P. H. Lu, B. Xiao, B. Hallam, B. Tjahjono, and S. Wenham, Sol. Energy Mater. Sol. Cells 130, 83-90 (2014), DOI: 10.1016/j.solmat.2014.06.037.

[33] G. Micard, Quantitative investigation of grain boundary recombination activity in multicrystalline silicon using light beam induced current contrast profiles, $\mathrm{PhD}$ Thesis, University of Konstanz (2011), http://nbn-resolving.de/urn: nbn:de:bsz:352-173272.

[34] G. Micard, G. Hahn, A. Zuschlag, S. Seren, and B. Terheiden, J. Appl. Phys. 108, 034516 (2010), DOI: 10.1063/1.3462447.

[35] FlexPDE 6.2, PDE Solutions Inc., 9408 E. Holman Rd., Spokane Valley, WA 99206, USA, http://www.pdesolutions. com. 